\title{
Evolutionary History of Triticum petropavlovskyi Udacz. et Migusch. Inferred from the Sequences of the 3-Phosphoglycerate Kinase Gene
}

\author{
Qian Chen ${ }^{1,29}$, Hou-Yang Kang ${ }^{19}$, Xing Fan ${ }^{1}$, Yi Wang ${ }^{1}$, Li-Na Sha ${ }^{1}$, Hai-Qin Zhang ${ }^{1}$, Mei-Yu Zhong ${ }^{1}$, \\ Li-Li Xu' ${ }^{1}$, Jian Zeng ${ }^{3}$, Rui-Wu Yang ${ }^{4}$, Li Zhang ${ }^{4}$, Chun-Bang Ding ${ }^{4}$, Yong-Hong Zhou ${ }^{1,2 *}$
}

1 Triticeae Research Institute, Sichuan Agricultural University, Sichuan, People's Republic of China, 2 Key Laboratory of Crop Genetic Resources and Improvement, Ministry of Education, Sichuan Agricultural University, Sichuan, People's Republic of China, $\mathbf{3}$ College of Resources and Environment, Sichuan Agricultural University, Sichuan, People's Republic of China, 4 College of Biology and Science, Sichuan Agricultural University, Sichuan, People's Republic of China

\begin{abstract}
Single- and low-copy genes are less likely to be subject to concerted evolution. Thus, they are appropriate tools to study the origin and evolution of polyploidy plant taxa. The plastid 3-phosphoglycerate kinase gene (Pgk-1) sequences from 44 accessions of Triticum and Aegilops, representing diploid, tetraploid, and hexaploid wheats, were used to estimate the origin of Triticum petropavlovskyi. Our phylogenetic analysis was carried out on exon+intron, exon and intron sequences, using maximum likelihood, Bayesian inference and haplotype networking. We found the $D$ genome sequences of Pgk-1 genes from $T$. petropavlovskyi are similar to the D genome orthologs in T. aestivum, while their relationship with Ae. tauschii is more distant. The A genome sequences of $T$. petropavlovskyi group with those of $T$. polonicum, but its Pgk-1 B genome sequences to some extent diverge from those of other species of Triticum. Our data do not support for the origin of T. petropavlovskyi either as an independent allopolyploidization event between Ae. tauschii and T. polonicum, or as a monomendelian mutation in T. aestivum. We suggest that T. petropavlovskyi originated via spontaneous introgression from T. polonicum into $T$. aestivum. The dating of this introgression indicates an age of 0.78 million years; a further mutation event concerning the $B$ genome occurred 0.69 million years ago.
\end{abstract}

Citation: Chen Q, Kang H-Y, Fan X, Wang Y, Sha L-N, et al. (2013) Evolutionary History of Triticum petropavlovskyi Udacz. et Migusch. Inferred from the Sequences of the 3-Phosphoglycerate Kinase Gene. PLoS ONE 8(8): e71139. doi:10.1371/journal.pone.0071139

Editor: Tianzhen Zhang, Nanjing Agricultural University, China

Received May 17, 2013; Accepted July 1, 2013; Published August 19, 2013

Copyright: (C) 2013 Chen et al. This is an open-access article distributed under the terms of the Creative Commons Attribution License, which permits unrestricted use, distribution, and reproduction in any medium, provided the original author and source are credited.

Funding: This work was financially supported by the National Natural Science Foundation of China (31101151), the National Transgenic Major Project (2011zx08009001), the National High-tech R\&D Program of China (863 Program) (2011AA100103-02), the Special Fund for Agro-Scientific Research in the Public Interest of China (201003021), and the Science and Technology Bureau and Education Bureau of Sichuan Province, China. The funders had no role in study design, data collection and analysis, decision to publish, or preparation of the manuscript.

Competing Interests: The authors have declared that no competing interests exist.

*E-mail: zhouyh@sicau.edu.cn

9 These co-first authors contributed equally to this work

\section{Introduction}

In the Xinjiang region of China, Triticum species are abundant. The Xinjiang rice wheat (Triticum petropavlovskyi Udacz. et Migusch.), known as 'Daosuimai' or rice-head wheat, is one of the Chinese endemic wheat landraces, together with the Sichuan white wheat complex (T. aestivum L.), Tibetan weedrace (T. aestivum ssp. tibetanum Shao) and Yunnan hulled wheat (T. aestivum ssp. yunnanense King) [1]. Based on chromosome pairing, morphology, eco-geographical origin and RFLP analysis, the Xinjiang rice wheat is distinct from other Chinese endemic wheat landraces [25].

The origin of $T$. petropavlovskyi has been discussed for decades. Gorsky [6] analyzed the morphology of Xinjiang rice wheat, and suggested that it was a mutated form of the tetraploid Triticum polonicum L.. However, Udachin and Miguschova [7] discovered that the Xinjiang rice wheat is not tetraploid but hexaploid, and named it $\mathcal{T}$. petropavlovskyi Udacz. et Migusch. The chromosomal constitutions of the Xinjiang rice wheat is AABBDD [2,8-10]. Dorofeev et al. [11] hypothesized that T. petropavlorskyi could be the result of spontaneous hybridization between $T$. aestivum and $T$. polonicum. Several previous studies indicated that the genes supporting a long glume in T. polonicum and T. petropavlovskyi were allelic and located on the long arm of chromosome 7A [12-14], agreeing with the hypothesis of spontaneous hybridization. Yen et al. [15] studied the natural distribution in Xinjiang of Aegilops tauschii, and Yang et al. [16] and Liu et al. [17] reported a similar distribution for a dwarfing accession of $T$. polonicum and suggested that T. petropavlovskyi is derived from a hybridization event between T. polonicum and Ae. tauschii. However, Efremova et al. [18] maintained that $T$. petropavlovskyi originated from $T$. aestivum through spontaneous mutation.

Despite prior intensive research, the origin of $T$. petropavlovskyi is still uncertain, and three hypotheses have been proposed: (1) T. petropavlovskyi is an independent species is derived from a natural hybridization event between T. polonicum and Ae. tauschii $[10,15,19$ 21]; (2) T. petropavlovskyi is a natural cross or backcross between $T$. polonicum and T. aestivum [2,11,12,22,23]; and (3) T. petropavlovskyi is a monogenic mutant of $T$. aestivum $[18,24]$. In a recently study, Kang et al. [25] created the synthetic hexaploid wheat (SHWDPW) between T. polonicum from Xinjiang and Ae. tauschï: its spike 
Table 1. Plants used in this study.

\begin{tabular}{|c|c|c|c|c|c|}
\hline Species & Genome & Accession & Origin & Abbrev. & GenBank Ac. No. \\
\hline Triticum urartu Thum. ex Gandil. & $A^{u}$ & TA763 & Lebanon & TUR63A & AF343474 \\
\hline Aegilops bicornis (Forskal) Jaub. et Spach. & $S^{b}$ & TA1954 & Egypt & AEB954S & AF343485 \\
\hline Aegilops longissima Schweinf. et Muschl. & $S^{\prime}$ & TA1912 & Israel & AEL912S & AF343487 \\
\hline Aegilops searsii Feldman et Kislev & $S^{s}$ & TA2355 & Israel & AES355S & AF343489 \\
\hline Aegilops sharonensis Eig & $\mathrm{s}^{\mathrm{sh}}$ & TA2065 & Turkey & AES065S & AF343486 \\
\hline Aegilops speltoides Tausch & S & TA2368 & Turkey & AES368S & AF343483 \\
\hline Aegilops speltoides var. ligustica (Savign.) Fiori & $\mathrm{s}$ & TA1770 & Iraq & AEL770S & AF343484 \\
\hline \multirow[t]{2}{*}{ Aegilops tauschii Cosson } & D & AS60 & Middle East & AET60D & $J Q 327050$ \\
\hline & & TA1691 & Unkown & AET691D & AF343479 \\
\hline \multirow[t]{6}{*}{ Triticum polonicum L. } & $A B$ & AS302 & Xinjiang, China & TPO302A & JQ327101 \\
\hline & & & & ТРО302B & JQ327102 \\
\hline & & AS304 & Xinjiang, China & TPO304A & JQ327088 \\
\hline & & & & TPO304B & JQ327089 \\
\hline & & PI42209 & Australia & TPO209A & JQ327096 \\
\hline & & & & TPO209B & JQ327097 \\
\hline \multirow[t]{4}{*}{ Triticum turgidum L. } & $A B$ & AS2233 & Xinjiang, China & TUR233A & JQ327113 \\
\hline & & & & TUR233B & JQ327114 \\
\hline & & AS2277 & Xinjiang, China & TUR277A & JQ327077 \\
\hline & & & & TUR277B & JQ327078 \\
\hline \multirow[t]{2}{*}{ Triticum durum Desf. } & $A B$ & AS2349 & Xinjiang, China & TDU349A & JQ327115 \\
\hline & & & & TDU349B & JQ327116 \\
\hline \multirow[t]{2}{*}{ Triticum durum Desf. cv. Langdon } & $A B$ & LDN & USA & TDULA & JQ327057 \\
\hline & & & & TDULB & JQ327058 \\
\hline \multirow[t]{4}{*}{ Triticum turanicum Jakubz. } & $A B$ & AS2229 & Xinjiang, China & TTU229A & JQ327109 \\
\hline & & & & TTU229B & JQ327110 \\
\hline & & AS2279 & Xinjiang, China & TTU279A & JQ327111 \\
\hline & & & & TTU279B & JQ327112 \\
\hline \multirow{4}{*}{$\begin{array}{l}\text { Triticum dicoccoides (Koern. ex Aschers. et } \\
\text { Graeb.) Schweinf. }\end{array}$} & $A B$ & TA51 & Israel & TDI51A & AF343481 \\
\hline & & & & TDI51B & AF343476 \\
\hline & & AS838 & Xinjiang, China & TDI838A & JQ327075 \\
\hline & & & & TDI838B & JQ327076 \\
\hline \multirow[t]{4}{*}{ Triticum carthlicum Nevski (syn. T. persicum Vav.) } & .) $A B$ & PI532494 & Kars, Turkey & TCA494A & JQ327065 \\
\hline & & & & TCA494B & JQ327066 \\
\hline & & PI532509 & Xinjiang, China & TCA509A & JQ327073 \\
\hline & & & & TCA509B & JQ327074 \\
\hline \multirow[t]{4}{*}{ Triticum timopheevii (Zhuk.) Zhuk. } & AG & TA2 & Armenia & $\pi T 2 A$ & AF343477 \\
\hline & & & & $\mathrm{TTI2G}$ & AF343488 \\
\hline & & Pl94761 & Georgia, USA & TI761A & JQ327126 \\
\hline & & & & TI761G & JQ327127 \\
\hline \multirow[t]{9}{*}{ Triticum petropavlovskyi Udacz. et Migusch. } & ABD & AS358 & Xinjiang, China & TPE358A & JQ327090 \\
\hline & & & & TPE358B & JQ327091 \\
\hline & & & & TPE358D & JQ327092 \\
\hline & & AS359 & Xinjiang, China & TPE359A & JQ327103 \\
\hline & & & & TPE359B & JQ327104 \\
\hline & & & & TPE359D & JQ327105 \\
\hline & & AS360 & Xinjiang, China & TPE360A & JQ327106 \\
\hline & & & & TPE360B & JQ327107 \\
\hline & & & & TPE360D & JQ327108 \\
\hline Triticum aestivum L. ssp. tibetanum Shao & $A B D$ & AS1026 & Xizang, China & ТТВ1026A & JQ327123 \\
\hline
\end{tabular}


Table 1. Cont.

\begin{tabular}{|c|c|c|c|c|c|}
\hline Species & Genome & Accession & Origin & Abbrev. & GenBank Ac. No. \\
\hline & & & & ТТВ1026B & JQ327124 \\
\hline & & & & TTB1026D & JQ327125 \\
\hline & & AS1027 & Xizang, China & ТТВ1027A & JQ327062 \\
\hline & & & & ТТВ1027B & JQ327063 \\
\hline & & & & ТTB1027D & JQ327064 \\
\hline \multirow[t]{9}{*}{ Triticum aestivum L. ssp. yunnanense King } & $A B D$ & AS331 & Yunnan, China & TYU331A & JQ327131 \\
\hline & & & & TYU331B & JQ327132 \\
\hline & & & & TYU331D & JQ327133 \\
\hline & & AS338 & Yunnan, China & TYU338A & JQ327085 \\
\hline & & & & TYU338B & JQ327086 \\
\hline & & & & TYU338D & JQ327087 \\
\hline & & AS343 & Yunnan, China & TYU343A & JQ327128 \\
\hline & & & & TYU343B & JQ327129 \\
\hline & & & & TYU343D & JQ327130 \\
\hline \multirow[t]{6}{*}{ Triticum sphaerococcum Perciv. } & $A B D$ & PI70711 & Iraq & TSP711A & JQ327117 \\
\hline & & & & TSP711B & JQ327118 \\
\hline & & & & TSP711D & JQ327119 \\
\hline & & PI115818 & Punjab, India & TSP818A & JQ327093 \\
\hline & & & & TSP818B & JQ327094 \\
\hline & & & & TSP818D & JQ327095 \\
\hline \multirow[t]{3}{*}{ Triticum macha Dekapr. et Menabde. } & $A B D$ & PI278660 & UK & TMA660A & JQ327082 \\
\hline & & & & TMA660B & JQ327083 \\
\hline & & & & TMA660D & JQ327084 \\
\hline \multirow[t]{6}{*}{ Triticum spelta $\mathrm{L}$. } & $A B D$ & PI347852 & Switzerland & TPL852A & JQ327098 \\
\hline & & & & TPL852B & JQ327099 \\
\hline & & & & TPL852D & JQ327100 \\
\hline & & PI347858 & Switzerland & TPL858A & JQ327120 \\
\hline & & & & TPL858B & JQ327121 \\
\hline & & & & TPL858D & JQ327122 \\
\hline \multirow[t]{6}{*}{ Triticum compactum Host } & $A B D$ & PI124298 & Unknown & TCO298A & JQ327070 \\
\hline & & & & TCO298B & $J Q 327071$ \\
\hline & & & & TCO298D & JQ327072 \\
\hline & & PI352299 & Switzerland & TCO299A & JQ327067 \\
\hline & & & & TCO299B & JQ327068 \\
\hline & & & & TCO299D & JQ327069 \\
\hline \multirow[t]{3}{*}{ Triticum aestivum L. cv. Chinese Spring } & $A B D$ & CS & Sichuan, China & TCHSA & JQ327051 \\
\hline & & & & TCHSB & JQ327052 \\
\hline & & & & TCHSD & JQ327053 \\
\hline \multirow[t]{3}{*}{ Triticum aestivum L. cv. Chuannong-16 } & $A B D$ & CN16 & Sichuan, China & TCN16A & $J Q 327054$ \\
\hline & & & & TCN16B & $J Q 327055$ \\
\hline & & & & TCN16D & $J Q 327056$ \\
\hline \multirow[t]{3}{*}{ Triticum aestivum L. cv. J-11 } & $A B D$ & $J-11$ & Sichuan, China & TJ11A & JQ327079 \\
\hline & & & & TJ11B & $J Q 327080$ \\
\hline & & & & TJ11D & JQ327081 \\
\hline \multirow[t]{3}{*}{ Synthetic hexaploid wheat } & $A B D$ & SHW-DPW & & SHWDA & $J Q 327059$ \\
\hline & & & & SHWDB & $J Q 327060$ \\
\hline & & & & SHWDD & JQ327061 \\
\hline Psathyrostachys juncea (Fischer) Nevski & Ns & PI222050 & Afghanistan & PJU050N & FJ711031 \\
\hline
\end{tabular}

The Genebank with AF numbers are from Huang et al. [25], those with JQ numbers have been assinged in this study.

doi:10.1371/journal.pone.0071139.t001 
Table 2. Parameters derived from $P g k-1$ sequence alignements.

\begin{tabular}{lllll}
\hline & & & & \\
\hline & Total sites & Variable characters & Conversed characters & Informative characters \\
\hline Exon+Intron & 1466 & 174 & 1292 & 91 \\
Exon & 894 & 88 & 806 & 36 \\
Intron & 572 & 107 & 378 & 80 \\
\hline doi:10.1371/journal.pone.0071139.t002 & & &
\end{tabular}

morphology was similar to T. petropavlovskyi. However, a comparison of SHW-DPW to T. petropavlorskyi, T. polonicum and related species by the phylogenetic analysis of the $A c c-1$ gene indicated that T. petropavlovskyi originated from the cross between T. polonicum from Xinjiang and exotic landraces of T. aestivum [26]. This finding contradicts the morphology-based conclusion of preceding study. Previous works based on different methods, including cytology, morphology and nuclear markers, have failed in identifying the origin of T. petropavlovskyi. Furthermore, no molecular-clock has been reported to examine the timing of its origin.

Single- and low-copy nuclear genes, being less susceptible to concerted evolution [27-29], are useful in phylogenetic study [3033 ] as well as in the identification of parents of allopolyploidy taxa [26,34-36]. Genes such as acetyl-CoA carboxylase 1 (Acc-1) [30], disrupted meiotic cDNA 1 (DMC1) and translation elongation factor $\mathrm{G}(E F-G)$ [37] have been particularly useful in elucidating the phylogenesis of Triticum-Aegilops species. The plastid 3phosphoglycerate kinase (PGK) gene, $P g k-1$, is a single copy nuclear gene in diploid species of the Triticeae; it is frequently considered to be superior to the $A c c-1$ gene for assessing the evolutionary history of polyploid wheats, because the $P g k-1$ gene has more parsimony informative sites than the $A c c-1$ gene $[31,38]$. For allotetraploid and allohexaploid species with two or three copies of genes present as single copies in diploid ancestors, the $P g k-1$ gene can both elucidate the phylogenetic relationships of such polyploid as well as potential progenitors [30,31,39,40].

In this study, we sequenced and analyzed the single-copy nuclear Pgk-1 gene in the following taxa: T. petropavlorskyi, SHWDPW (synthetic hexaploid wheat between T. polonicum and Ae. tauschii), and the hypothetical Triticum and Aegilops progenitors of T. petropavlovskyi to reveal their phylogenetic relationships and to explore both the origin of T. petropavlovskyi and its divergence time from other taxa.

\section{Materials and Methods}

\section{Plant materials}

The species, genomic constitutions, origin, GenBank accessions, and sources of the taxa are listed in Table 1. The sequences of $p g k-$ 1 gene of the accensions with TA numbers were obtained from the GenBank database; the rest of the species considered for the sequences are reported here for the first time.

The accessions with PI and AS numbers were kindly provided by the American National Plant Germplasm System (Pullman, Washington, USA) and the Triticeae Research Institute, Sichuan Agricultural University, China, respectively. The artificial synthetic amphiploid of Triticum polonicum and Aegilops tauschii (SHWDPW) was produced by Kang et al. [25]. The plants and voucher specimens have been deposited at Herbarium of Triticeae
Research Institute, Sichuan Agricultural University, China (SAUTI).

\section{DNA extraction, amplification and sequencing}

DNA was extracted from fresh leaves of single plants, following a standard CTAB (cetyltrimethylammonium bromide) protocol [41]. For amplification of the $P g k-1$ gene, a pair of $P g k 1$-specific primers, PPF1 (5'-CACGTGGGTCGTCGTAAGGGTGTT-3') and PPR1 (5'-ACGACGAGTTGTGTTGTGGCTCAT-3'), was used [31]. Polymerase chain reactions (PCR) were performed in a GeneAmp 9700 Thermal Cycler (Applied Biosystems Inc., California, USA) according to the following cycling program: initial denaturation at $94^{\circ} \mathrm{C}$ for $5 \mathrm{~min} ; 35$ cycles of $94^{\circ} \mathrm{C}$ for $30 \mathrm{~s}$, $56^{\circ} \mathrm{C}$ for $30 \mathrm{~s}, 68^{\circ} \mathrm{C}$ for $5 \mathrm{~min}$; followed by a final elongation period at $68^{\circ} \mathrm{C}$ for $10 \mathrm{~min}$. A final volume of $50 \mu \mathrm{l}$ for each PCR reaction was prepared, containing $0.5 \mu \mathrm{g}$ of genomic DNA, $10 \times$ reaction buffer, $1.5 \mathrm{mM}$ of each primer, $2.5 \mathrm{mM}$ of each dNTP, $2.5 \mathrm{mM} \mathrm{MgCl} 2,2$ units of high-fidelity ExTaq DNA polymerase (Takara Biotechnology Co. Ltd., Dalian, China).

$1.0 \%$ agarose gel was used to estimate the size of the amplification products, which were purified using the EZNATN gel extraction kit (Omega Bio-Tech, Georgia, USA) and stored in $30 \mu \mathrm{l}$ TE buffer. The purified products were cloned into the pMD19-T vector (Takara) according to the manufacturer's instructions. Cloning of PCR amplifications from single-copy nuclear genes from allopolyploid species should isolate homoeologous sequences from each nuclear genome [42,43]. For the hexaploid Triticum species, the A, B and D genomes homoeologous sequences of $P g k-1$ gene were isolated, and the A and B genomes homoeologous sequences were separated for the tetraploid Triticum. The cloned PCR products were commercially sequenced on both strands by the Beijing Genomics Institute (BGI, Shenzhen, China). All the sequences used in the phylogenetic analysis were derived from at least five independent clones.

\section{Alignments and phylogenetic analysis}

Multiple sequences were aligned using Clustal X with default parameters, followed by manual adjustment to minimize gaps [44]. In an initial phylogenetic analysis, if all sequences used for alignment derived from independent clones, formed a monophyletic group, then only one sequence was used later on. Distinct sequences derived from single accessions mapping different clades were all included in the phylogenetic analysis. Nucleotide frequencies, transition/transversion ration, and variability in different regions of the sequences were examined by MEGA 5.0 [45].

Three data matrixes, including exon+intron data (the target $P g k$ 1 gene sequences), exon data and intron data, were used separately to implement phylogenetic analyses. Phylogenetic trees were 
B-T. dicaccoides (TA51)

B-T. durum cv. Langdon (LDN)

B.T. destoum ssp.jzonnanense (AS331)

B-T. carthlicum (P1532494) (AS343)

B-T, polonicum (PI42209)

B-T. aestivum ssp. tibetamum (AS1026)

B-T. sphacrocaccum (PI
B-T. spelta (P1347858)

B-T. aestivum ssp. yumnanense (AS338)

B-T. carthlicum (PI532509)

B-Synthetic hexaploid wheat (SHW-DPW)

B-T. polonicum (AS302)

B-T. polonicum (AS302)
B-T. turanicaum (AS2229)

-T. turanictum (AS22

B.T. durum (AS2349)

B-T. macha (P1278660)

B-T. turgidum (AS2277)
B-T turgidum (AS2233)

B-T. compactum (P1352299)

B-T. compactum (PI124298)

B-T. aestivum cv. Chuannong-16 (CN16)

B-T. dicoccoides (AS838)

B-T. aestivum ssp. tibetanum (AS1027)

B-T. aestivum ev. Chinese Spring (CS)

B-T. petropanlovskyi (AS359)

B-T petropavlosshyi (AS358)

B-T. sphaerococcum (P170711)

G-T. timopheevii (TA2)

Ae. searsii (TA2355)

S-Ae. bicornis (TA1954)

S-Ae, sharonensis (TA2065)
S-Ae. longissima (TA1912)

S-Ae, speltoides var. ligustica (TA1770)

$\mathrm{S}-$ Ae. speltoides $(\mathrm{TA} 23 \mathrm{~S})$

D-Ae. tauschii (AS60)

D-Synthetic hexaploid wheat (SHW-DPW)

D-T. aestivum cv. Chuannong-16 (CN16)

D-T. compactum (PI352299)

D-T. sphaerococcum (P170711)

D-T. sphaerococcum (P170711)

D-T. spelta (P1347858)

D-T. aestivum ssp. tibetamum (AS1027)
D-T. aestivum ssp. tibetamum (AS1026)

D-T. petropanlovskivi (AS360)

D-T. aestivum ssp. yumnanense (AS343)

D-T. petropaviouskyi (AS359)
D.T. petroparlowxky (AS358)

D-T, spelta (P1347852)

D-Ae. tauschiii (TA1691)

D-T. aestivum cv. Chinese Spring (CS)

-T. aestivum ssp. yunnanense (AS331)

D-T. macha (P1278660)

A-T. durum (AS2349)

A-T. polonicum (P142209)

A-T. polonicum (PI422

A-T. turanicum (AS2279)

A.T.

A-T. timopheevii (P194761)

A-T. turgidum (AS2233)

A-T. petropastovshyi (AS358)

A-T. spelia (PI347852)

A-T. polonicum (AS302)

A-T. dicoccoides (AS838)

A.T. aestivum cy. Chuannong-16 (CN16)

A-T

A-T. curanicum (AS229)

A-T. dicoccoides (TAS1)

A-T. sphacrococcum (PI70711)

A-T. aestivum cv. J-11 (J-11)

A-T. aestivum ssp. yunnanense (AS343)

A-T. petropavlovskvi (AS359)

A-T. sphaerococcum (PII15818)
A-T. aestinum ssp. tibetamum (AS1026)

A-T. carthlicum (PIS32494)

A-T. aestivum ssp. yzunnanense (AS331)

A-T. petropaslovskyi (AS360)

A-T. carthlicum (PI532509)

A-T. aestivum ssp. tibetanum (AS1027)
A-T. aestivem ev, Chinese Spring (CS)

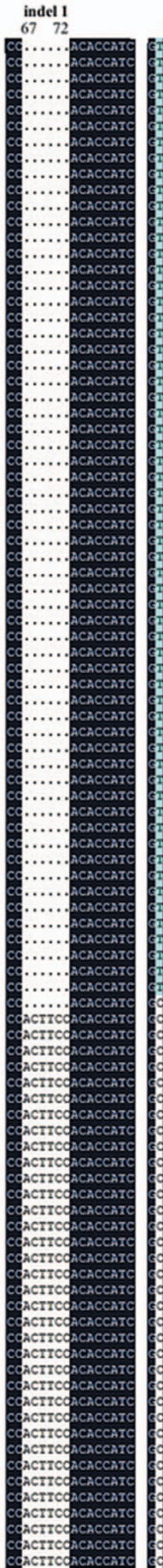

indel 2

1308

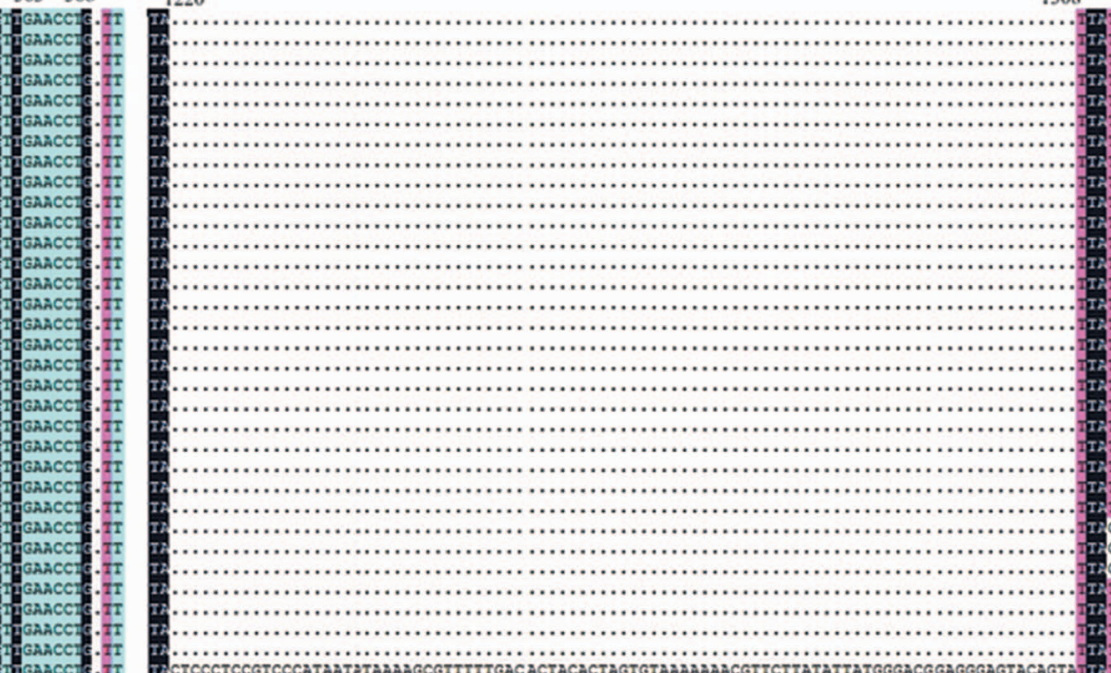

-

Figure 1. Maximum-likelihood tree from the exon+intron sequences of the Pgk-1 gene of $T$. petropavlovskyi and its related species. Numbers above nodes are bootstrp values $>50 \%$ numbers below nodes are posterior probability values $>90 \%$. Genome composition, species name and accession number/cultivar name are indicated for each taxon.

doi:10.1371/journal.pone.0071139.g001 
T. petropavlovskyi

T. polonicum

synthetic hexaploid wheat

Ae. tauschii

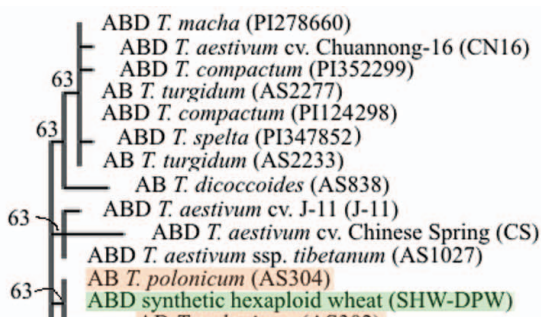

ABD T. macha (PI278660)

ABD T aestivum cv. Chuannong-16 (CN16) ${ }_{63}$ ABD T. compactum (P1352299)

ABD T. Caun (AS2277)

63 (

AB T. turgidum (AS2233)

AB T. turgidum (AS2233)

63- $\mathrm{ABD}$ T. aestivum cv. J-11 (J-11)

- ABD T. aestivum cv. Chinese Spring (CS)

ABD T. aestivum ssp. tibetanum (AS1027)

63- $\mathrm{AB}$ T. polonicum (AS304) ABD synthetic hexaploid wheat (SHW-DPW)

- AB T. polonicum (AS302)

86 AB T. turanicum (AS2229)

98 AB T turanicum (AS2279)

88 ABD $T$ spelta (PI347858)

- AB T. durum cv. Langdon (LDN)

- AB T. carthlicum (PI532495)

AB $T$. dicoccoides (TA51)

- AB 1. carthlicum (PI532509)

91 - AB T. polonicum (PI42209)

91 ABD T. sphaerococcum (PI115818)

- $\mathrm{AB}$ T. durum (AS2349)

- ABD T. aestivum ssp. yunnanense (AS338)

ABD T. aestivum ssp. yumnanense (AS331)

ABD $T$ aestivum ssp. tibetamum (AS1026)

71- ABD T. petropavlovskyi (AS359)

ABD T. petropavlovskyi (AS358)

ABD T. petropavlovskyi (AS360)

- ABD T. sphaerococcum (PI70711)

- ABD T. sphaerococcum (PI70711)

ABD T. aestivum cv. Chinese Spring (CS)

- ABD T. aestivum ssp. tibetanum (ÂS1027)

ABD T. aestivum cv. J-11 (J-11)

D Ae. tauschii (TA1691)

ABD T. spelta (PI347852)

- ABD T. sphaerococcum (PI115818)

ABD T. petropavlovskyi (AS358)

- ABD T. petropavlovskyi (AS360)

ABD $T$. petropavlovskvi (AS359)

ABD T. aestivum ssp. yunnanense (AS338)

- ABD T. aestivum ssp. yunnanense (AS331)

ABD T. aestivum ssp. yunnanense (AS343)

79 - ABD T. aestivum ssp. tibetanum (AS1026)

87 ABD T. spelta (P1347858)

$87 \mathrm{D}$

100 ABD synthetic hexaploid wheat (SHW-DPW)

ABD T. compactum (PI352299)

ABD T. aestivum cv. Chuannong-16 (CN16)

ABD T. compactum (PI124298)

ABD T. macha (PI278660)

$100-\mathrm{S}$ Ae. speltoides var. ligustica (TA1770)

$100 \quad \mathrm{~S}$ Ae. speltoides (TA2368)

$100-$ AG T. timopheevii (TA2)

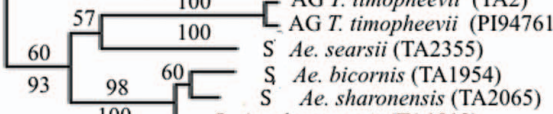

$100 \quad S$ Ae. sharonensis (TA1912)

${ }^{64} \mathrm{AB}$ T. durum (AS2349)

AB T. turgidum (AS2277)

$\mathrm{AB} T$. durum cv. Langdon (LDN)

ABD $T$ aestivum cv. Chuannong-16 ( $\mathrm{CN} 16)$

ABD T. compactum (PI352299)

AB $T$. dicoccoides (AS838)

AB $T$ dicoccoides (TA51)

- AB T. carthlicum (PI532509)

95- ABD $T$. compactum (PI124298)

AB T. polonicum (AS302)

ABD T. spelta (PI347852)

ABD T. spelta (Pl347852) 4209 )

ABD T. petropavlovskyi (AS358)

$\mathrm{AB}$ T. turgidum (AS2233)

- AB T. turanicum (AS2279)

u AB T. turanicum (AS2229)

- A T. urartu (TA763)

AG T. timopheevii (PI94761)

AG T. timopheevii (TA2)

ABD $T$. aestivum ssp. tibetanum (AS1026)

ABD T. aestivum ssp. tibetanum (AS1027)

ABD synthetic hexaploid wheat (SHW-DPW)

AB T. carthlicum (PI532495)

- ABD T. aestivum cv. J-11 (J-11)

ABD T. aestivum cv. J-11 (J-11)
ABD T. sphaerococcum (PI115818)

$90 \quad-\quad$ AB T polonicum (AS 304$)$

100 - ABD T. sphaerococcum (PI70711)

ABD T. petropavlovskyi (AS360)

ABD T. petropavlovskyi (AS359)

- ABD T. aestivum ssp. yunnanense (AS338)

- ABD T. aestivum ssp. yunnanense (AS331)

ABD T. aestivum ssp. yumnanense (AS343)

ABD T. macha (PI 278660$)$

ABD T. aestivum cv. Chinese Spring (CS)

Ns Psathyrostachys juncea (PI222050)

0.0 
Figure 2. Maximum-likelihood tree from the exon+intron sequences of the Pgk-1 gene of T. petropavlovskyi and its related species. Numbers above nodes are bootstrp values $\geq 50 \%$ numbers below nodes are posterior probability values $\geq 90 \%$. Genome composition, species name and accession number/cultivar name are indicated for each taxon. doi:10.1371/journal.pone.0071139.g002

created using Maximum likelihood (ML) and Bayesian inference (BI). ML analysis was carried out with PAUP*4.0b10 (Swofford, D. L., Sinauer Associates, http://www.sinauer.com), and Psathyrostachys juncea (Fischer) Nevski was used as an outgroup. The best-fit models of sequence evolution for ML analysis were estimated using ModelTest v3.0 with Akaike information criteria (AIC) [46]. The optimal models were found to be $\mathrm{HKY}+\mathrm{G}$ for the exon+intron data, TVM+G for the intron data, and $\mathrm{TrN}+\mathrm{G}$ for exon data. ML heuristic searches were performed with 100 random addition sequence replications and TBR branch swapping algorithm. The robustness of the trees was estimated using bootstrap support (BS) [47]. ML bootstrapping was performed with 250 replicates, each with three replicates of stepwise random taxon addition, using the same model and parameters. BS-values under $50 \%$ were not included in figures.

Bayesian inference (BI) analysis was performed using MrBayes v3.2 [48] with Psathyrostachys juncea used as an outgroup. The bestfit models for BI analysis were carried out with AIC using MrModelTest v2.3 (http://www.ebc.uu.se/systzoo/staff/ nylander.html). The optimal models were found to be $\mathrm{GTR}+\mathrm{I}+\mathrm{G}$ for exon+intron data, GTR $+\mathrm{G}$ for intron data and exon data. Four MCMC (Markov Chain Monte Carlo) chains (one cold and three heated) were applied with default setting. In order to make the standard deviation of split frequencies fall below 0.01, 4,200,000 generations for exon+intron data, 3,000,000 generations for intron data, and 5,000,000 for exon data were run. Samples were taken every 100 generations under the best-fit model. For all analyses, the first $25 \%$ of samples from each run were discarded as "burn-in" to ensure the stationarity of the chains. Bayesian posterior probability $(\mathrm{PP})$ values were obtained from a majority rule consensus tree generated from the remaining sampled trees. PP-values less than 90\% were not included in figures.

\section{Network analysis}

The median-joining (MJ) network method [49], and Templeton, Crandall and Sing (TCS) method [36], have been shown to be effective methods for revealing specific progenitor descendant relationships of perennial Triticeae [21,50-52], and were thus performed in this study. Before reconstructing the MJ and TCS networks, a test of recombination was performed using the Phi (Pairwiser homoplasy index) method within Splits tree [53]. Building upon this test, the sequences of A $(\mathrm{P}=0.64932), \mathrm{B}$ $(\mathrm{P}=0.9999)$ and $\mathrm{D}$ genomes $(\mathrm{P}=1.0)$ were used to generate the MJ network, and the exon data $(\mathrm{P}=0.7493)$ was used to generate the TCS network. MJ network analysis was generated by Network 4.1.1.2 program (Fluxus Technology Ltd, Clare, Suffolk, UK), and TCS haplotype network was performed to evaluate possible genetic relationships between haplotypes with the computer program TCS 1.2.1 [54].

\section{Divergence dating}

The potential clock-like evolution of $P g k-1$ sequences was evaluated with a likelihood ratio rate comparing the likelihood scores from the unconstrained and clock-constrained analyses, implemented in PAUP*4.0b10. The molecular clock was rejected because the substitution rates were significantly heterogeneous $(\chi 2=154.90, \mathrm{df}=95, \mathrm{P}=0.0001)$, implying a very poor fit to the molecular clock. Therefore, divergence times with 95\% confidence intervals (C.I.) were estimated using the Bayesian relaxed molecular clock method, implemented in BEAST v1.7.1 [55].

The lack of fossils for Triticeae precluded a direct calibration of tree topologies. Instead, molecular dating of the intron data was estimated on the basis of the intron region of the $P g k-1$ gene clock of 0.0051 substitutions per site per MY (million year) [31,38], setting the clock for the divergence of Pooideae and Panicoideae sub-families at 60 MYA [56-58]. Calibration points were performed using a relaxed uncorrelated lognormal molecular clock. MCMC searches were run for 10,000,000 generations under GTR+G model (with the associated parameters specified by MrModelTest as the priors), with the first 2,000,000 discarded as burn-in. Trees were then viewed in FigTree vl.3.1 (http://tree. bio.ed.ac.uk/).

\section{Results}

\section{Sequence analysis}

In all polyploid species considered, the expected number of copies of the $P g k-1$ gene were successfully amplified. The DNA sequence of the $P g k-1$ gene includes 5 exons and 4 introns, which range in length from $1360 \mathrm{bp}$ to $1476 \mathrm{bp}$, as known from previous study $[30,31,38]$ (Table 2). The lengths of exon+intron, exon and intron data sets were 1466, 894 and $572 \mathrm{bp}$, respectively. As expected, the level of nucleotide variation in the exon region (88 variable sites and 36 parsimony-informative sites) was lower than that in the intron region (107 variable sites and 80 parsimonyinformative sites). The average content of $\mathrm{G}+\mathrm{C}$ of exon+intron, and exon was 42.9 , and $47.3 \%$, respectively, and the transition/ transversion ratio was 2.13 , and 2.24 , respectively. The alignment of the exon sequence was unambiguous and without gaps. Gaps were, on the contrary, present in introns. In particular, apart from single nucleotide substitutions and deletions, three significant indels (insertion/deletion) (indel 1, 2, 3) were found (Fig. 1). Indel 1 was located at position 67-72 of the A genome; indel 2 mapped at position 563-568 and had a 6 bp deletion specific for A genome. Unexpectedly, the T. aestivum ssp. yunnanense (AS338) did not show the indel 2. Indel 3 was presented at position 1220-1308 and had a $89 \mathrm{bp}$ insertion in the $\mathrm{G}$ genome of T. timopheevii.

\section{Phylogenetic analyses}

Using Psathyrostachys juncea as an outgroup, the three data sets corresponding to exon+intron, exon and intron were used phylogenetic analyses (ML and BI) were carried out. ML analysis of the exon+intron data generated a single phylogenetic tree ( - Lnlikelihood $=4092.85$ ), with the following parameters: $\mathrm{A}=0.26 ; \mathrm{C}=0.21 ; \mathrm{G}=0.23, \mathrm{~T}=0.30$, gamma shape parameter $=0.29$. Bayesian analysis of the same data recovered the same topology. In Figure 2, the ML tree is reported with values of the bootstrap support (BS) above and posterior probabilities (PP) below branches.

The ML tree of Figure 2 indicates that all homoeologous Pgk-1 sequences from polyploid accessions are grouped with those of the 
T. petropavlovskyi $\quad \mathbf{9 8}\left[\begin{array}{l}\text { AG } T \text {. timopheevii (TA2) } \\ \text { AG T. timopheevii (PI94761) }\end{array}\right.$

\section{T. polonicum}

synthetic hexaploid wheat

Ae. tauschii

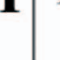

90

100

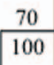

100

- ABD T. petropavlovskyi (AS358)

ABD T. petropavlovskyi (AS360)

ABD T. sphaerococcum (PI70711)

$\mathrm{S}^{\mathrm{S}}$ Ae. searsii (TA2355)

AB T. carthlicum (PI532509)

- ABD T. aestivum cv. Chuannong-16 (CN16)

AB T. polonicum (P142209)

ABD T. compactum (PI352299)

ABD T. compactum (PI124298)

AB T. dicoccoides (TA51)

AB T. turgidum (AS2277)

ABD $T$. aestivum ssp yumnanense (AS338)

- ABD T. sphaerococcum (PI115818)

$\mathrm{ABD}$ T. spelta (PI347852)

AB T. turgidum (AS2233)

ABD $T$. aestivum ssp. tibetanum (AS1026)

- ABD T. aestivum ssp. yunnanense (AS343)

[ ABD synthetic hexaploid wheat (SHW-DPW)

AB T. polonicum (AS304)

$100 \mathrm{AB}$ T polonicum (AS302)

90- AB T. turanicum (AS2279)

- $\mathrm{AB} T$. durum cv. Langdon (LDN)

- $\mathrm{AB}$ T dicoccoides (AS 838 )

- AB T. durum (AS2349)

[ ABD synthetic hexaploid wheat (SHW-DPW)

100. ABD synthetic hexaploid wheat (SH W-DP

- ABD T. compactum (PI352299)

85 - ABD T. compactum (PI124298)

98 - ABD T. sphaerococcum (PI70711)

- ABD $T$. aestivum ssp. tibetanum (AS1026),

D Ae, tauschii (TA1691)

ABD T. aestivum cv. Chinese Spring (CS)

ABD T. aestivum ssp. tibetamum (AS1027)

ABD T. aestivum cv. J-11 (J-11)

ABD $T$, petropavlovsky (AS358)

ABD T. spelta (PI347852

ABD $T$. petropavlovskyi AS 359 )

ABD T. petropavlovskyi (AS359)

ABD T. petropavlovskyi (A

- ABD T. aestivum ssp. yumnanense (AS343)

ABD T. sphaerococcum (PI115818)
ABD T. aestivum ssp. yunnanense (AS331)

ABD T. macha (PI278660)

ABD T. aestivum ssp. yunnanense (AS338)

AB T. polonicum ( $\mathrm{AS} 302)$

AB T. turanicum (AS2229)

- $\mathrm{AB}$ T. turanicum ( $\mathrm{AS} 22$

A T. Trartir(TA763)

- AB T. polonicum (PI42209)

- ABD T. aestivum cv. Chuannong-16 (CN16)

- AB T durum cv. Langdon (LDN)

- ABD T. compactum (PI352299)

ABD T. compactum (PI124298)

$\mathrm{AB} T$. dicoccoides (AS838

ABD T. petropavlovskyi (AS358)

ABD T. spelta (PI347852)

$\mathrm{AB}$ T. turgidum (AS2233)

AB T. durum (AS2349)

ABD T. spelta (P1347858)

AG T. timopheevii (TA2)

- AB T dicocoides (TA51)

$\angle$ AB $T$. carthlicum (PI532509)

ABD T. petropavlovskyi (AS360)

- ABD T. aestivum cv. J-11 (J-11)

- ABD T. petropavlovskvi (AS359)

ABD T. aestivum ssp. yunnanense (AS343)

78 - AB 1. polonicum (AS304)

$100-A B D$ synthetic hexaploid wheat (SHW-DPW)

- AB T. carthlicum (PI532494)

- ABD T. sphaerococcum (PI1 15818)

ABD T sphaerococcum (PI70711)

ABD T. aestivum ssp. tibetanum (ÂS1026)

- ABD T. aestivum ssp. yunnanense (AS331)

- ABD T. aestivum ssp. tibetamum (AS1027)

ABD T. macha (PI278660)

ABD $T$. aestivum cv. Chinese Spring (CS)

$92-\mathrm{S}$ Ae. speltoides var. ligustica (TA1170)

$100-\mathrm{S}$ Ae. speltoides (TA2368)

$100 \quad$ S Ae. longissima (TA1912)

$00-\mathrm{S}$ Ae. sharonensis (TA2065)

- Ns Psathyrostachys juncea (PI222050)
G

B

$\mathbf{S}$

D

$\mathbf{A}$ 
Figure 3. Bayesian tree inferred from the intron sequences of the Pgk-1 gene of $T$. petropavlovskyi and its related species. Numbers above nodes are bootstrap values $>50 \%$; below nodes are Bayesian posterior probability values $>90 \%$. Genome composition, species name and accession number/cultivar name are indicated for each taxon. doi:10.1371/journal.pone.0071139.g003

diploid parental species. The tree has two major clades: the one including sequences of the A genome and the second those derived from the genomes B, D, G and S. In Clade I, the A genome specific sequences from three T. petropavlovskyi accessions and from Triticum species (except T. aestivum cv. Chinese Spring) formed a group with $90 \%$ bootstrap value and $100 \%$ posterior probabilities support. One T. petropavlovskyi accession (AS358), together with two accessions of T. polonicum (AS304 and PI42209), formed a subclade, with bootstrap value of $95 \%$. In Clade II, the B genome sequences from three $T$. petropavlovskyi accessions clustered together in a well supported $(71 \%$ BS and 98\% PP) subclade. SHW-DPW had a topology contiguous with two accessions of T. polonicum (AS302 and AS304), with 63\% bootstrap support. The sequences from the D genomes mapped to two subclades. One consisted of three accessions of T. petropavlovskyi, eleven of T. aestivum and one of Ae. tauschii (TA1691), with 72\% bootstrap support. The second one included SHW-DPW and Ae. Tauschii (AS60) with 87\% BS and $100 \%$ PP.

ML analysis of the intron data yielded a single phylogenetic tree $(-$ Lnlikelihood $=1804.87$, with parameters: $\mathrm{A}=0.29 ; \mathrm{C}=0.18$; $\mathrm{G}=0.17 ; \mathrm{T}=0.36$ and gamma shape parameter $=0.60$. The Bayesian analysis generated the same topology, as illustrated in Figure 3. Two major clades are evident: Clade I includes only the Pgk-1 sequences from the $\mathrm{G}$ genome with a high bootstrap support (99\% BS, 98\% PP). The Clade II includes A, B, D and S genomes sequences and is congruent with the tree inferred from the exon+intron data, except for nodes presenting different statistical support. In Clade II, sequences from the B genome clustered together with a good support $(70 \%$ BS and $100 \%$ PP). In this B genome clade, with the exception of T. sphaerococcum (PI70711), all accessions were grouped in a well supported subclade $(92 \%$ BS and $100 \% \mathrm{PP}$ ); the three accessions of T. petropavlovskyi were separated from other Triticum. In the A genome subclade, $T$. petropavlovskyi (AS359) mapped together with T. polonicum (AS304) (78\% BS and 100\% PP).

Exon data generated a single ML phylogenetic tree (-Lnlikelihood $=2150.29)$, with the following parameters $\mathrm{A}=0.24$, $\mathrm{C}=0.21, \mathrm{G}=0.27, \mathrm{~T}=0.28$, gamma shape parameter $=0.38$. $\mathrm{ML}$ and BI analysis of the same data supported a similar topology. Figure 4 reports the ML tree of exon sequences which includes two major clades: Clade I, consisting of A genome sequences: T. carthlicum (PI532509) and T. petropavlovskyi (AS360) are mapped in the same group $(64 \% \mathrm{BS}$ and $95 \% \mathrm{PP})$. In the second clade (Clade II), which includes $\mathrm{B}, \mathrm{D}, \mathrm{G}$, and $\mathrm{S}$ genomes, the $\mathrm{B}$ genome sequences of three accessions of T. petropavlovskyi grouped together (64\% BS and 93\% PP), separated from other accessions. Ae. tauschii (TA1691) and Ae speltoides (TA2368) clustered in a subclade $(76 \%$ BS and $97 \% \mathrm{PP})$.

\section{Network analysis}

To highlight the relationships among haplotypes of the Pgk-1 sequence, network methods were employed and the exon+intron data (Fig. 5) and exon data (Fig. 6) were considered. In Figure 5, each circular network node represents a haplotype, with node size being proportional to number of its isolates. Mv (median vectors representing missing intermediates) shows unsampled nodes inferred from the MJ network analysis. The number on the branches indicates the positions of the mutations. Network loops represent either true reticulation events or alternative genealogies in closely related lineages. MJ analysis of the $P g k-1$ exon+intron data recovered groupings corresponding to clades revealed by ML phylogeny. T. petropavlovskyi, as expected, was present in three clusters (A, B and D), representing the A, B and D genomes. Most accessions of T. aestivum, except T. aestivum cv. Chinese spring, were included in the A-type. The A-type sequences of three accessions of $T$. petropavlorskyi were included in subgroups I and II. $T$. petropavlovskyi (AS358) and T. polonicum (AS302) were placed at a central branching point. Meanwhile, in the B-type cluster, three accessions of $T$. petropavlovskyi grouped together in subgroup III, and T. polonicum (AS304) together with SHW-DPW in subgroup IV. In the D-type cluster, T. petropavlouskyi (AS358 and AS359), T. aestivum cv. Chinese Spring, T. aestivum cv. J-11, T. aestivum ssp. yunnanense (AS343) and T. spelta (PI347852) resulted included in subgroup $\mathrm{V}$, while the sequences from the amphiploid SHW-DPW and Ae. tauschii formed the distinct subgroup VI. The TCS procedure [36] was used to illustrate haplotype relationships among accessions. TCS defined a 95\% parsimony connection limit of 13 steps for exon alignment of fifty haplotypes derived from 96 sequences (Fig. 6). The TCS network consisted of three major haplotypic groups corresponding to the A, B and D genomes. The length of the branches between two nodes was proportional to the nucleotidic difference. In TCS analysis, $\mathcal{T}$. petropavlovskyi (AS360) shows a close haplotype relationship with $\mathcal{T}$. carthlicum from Xinjiang, China, supporting the exon results of the ML and BI analyses. Two further differences between the TCS and MJ were noted. Firstly, the haplotype of the D genome of $T$. macha (PI278660) appeared related to haplotypes of the B genome accessions. Secondly, Ae tauschii (TA1691) showed a close haplotype relationship with the $\mathrm{S}$ genome of Ae. speltoides var. ligustica.

\section{Molecular dating}

The BEAST analysis of the intron region of $P g k-1$ was used to derive a time-calibrated phylogenetic tree (Fig. 7). Under a lognormal relaxed clock, rate variation was equal to $0.96(95 \%$ C.I., 0.67-1.39), supporting the adoption of the relaxed clock method. The Yule prior was equal to 0.47 (95\% C.I., 0.37-0.63) and five homoeologous types of the $P g k-1$ gene, A-, B-, D, G- and S-type, clustered in distinct clades. The divergence time of the A, $\mathrm{B}$, and D genomes of T. petropavlorskyi was estimated equal to 1.13 (95\% C.I., 0.65-1.75), 1.02 (95\% C.I., 0.24-1.51), and 0.73 MYA (95\% C.I., 0.41-1.01), respectively. The split between Pgk-1 A and D genomes of $\mathcal{T}$. petropavlovskyi and its putative diploid genome donor, T. polonicum and Ae. tauschii, took place around 0.74-1.13 and 0.33-0.73 MYA, respectively. The B genome diverged from the $\mathrm{S}$ genome at 2.27 MYA (95\% C.I., 1.68-3.19), while the divergence time of $T$. petropavlovskyi and $T$. polonicum was $0.68-0.91$ MYA for A genome. The divergence time of $\mathcal{T}$. petropavlovskyi and T. polonicum and the B genome was 0.34-0.78 MYA. The divergence time of $T$. petropavlovskyi from hexaploid wheat resulted equal to 0.14-0.33 MYA (A genome), 0.16-0.69 MYA (B genome) and 0.11-0.27 MYA (D genome), respectively (node 1-node 9). In 


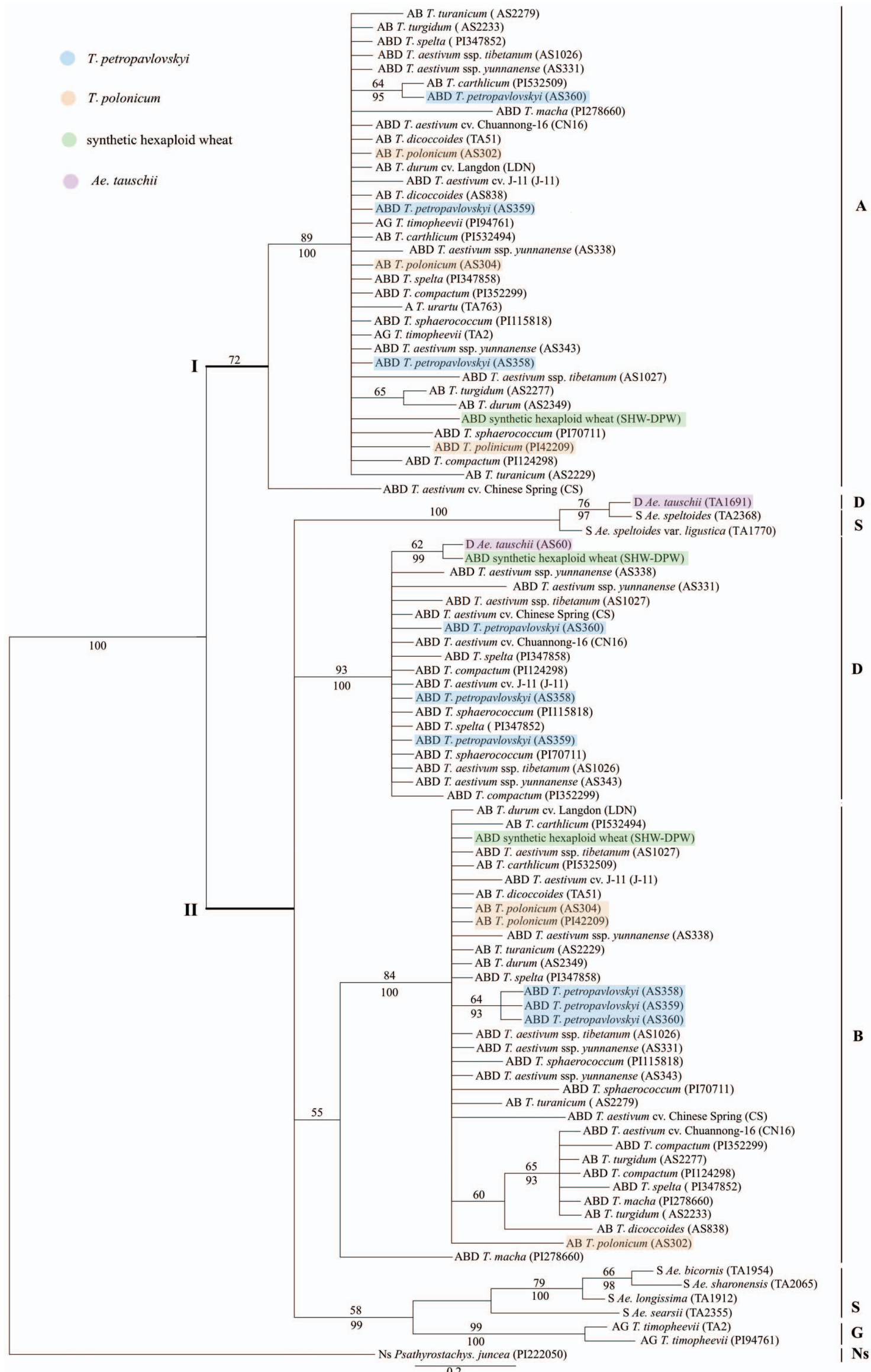


Figure 4. Maximum-likelihood tree inferred from the exon sequences of the Pgk-1 gene of $T$. petropavlovskyi and its related species. Numbers above nodes are bootstrap values $\geq 50 \%$; below nodes are Bayesian posterior probability values $\geq 90 \%$. Genome composition, species name and accession number/cultivar name are indicated for each taxon. doi:10.1371/journal.pone.0071139.g004

the tree, the tetraploid wheat $T$. polonicum diverged earlier than $T$. petropavlovskyi. In the A genome, the divergence time of $T$. petropavlovskyi (AS358) was later than the other two accessions of T. petropavlovskyi. On the contrary, in $\mathrm{B}$ and $\mathrm{D}$ genomes, the divergence time was earlier than the other two accessions. Additionally, the divergence time of B genome of T. petropavlovskyi was earlier than other three Chinese endemic wheat landraces. Between T. petropavlovskyi and SHW-DPW, a significant difference was observed: in the genomes $\mathrm{A}, \mathrm{B}$ and $\mathrm{D}$, the divergence time of SHW-DPW was later than T. petropavlovskyi.

\section{Discussion}

\section{Relationships between T. petropavlovskyi and hexaploid wheat taxa}

Based on cytological results, Yao et al. [3] and Chen et al. [23] suggested that the B genome was responsible for the difference between T. petropavlovskyi and T. aestivum cv. Chinese Spring, and that two pairs of chromosomes, one identified as chromosome $6 \mathrm{~B}$ [2], were involved. The allelic variation at the HMW glutenin subunits loci, Gli-1 and Gli-2, supported the cytological results [59]. Also, Yang et al. [10] reported that T. petropavlovskyi differed from T. spelta in at least one or two pairs of chromosomes. Results based on molecular markers, including A-PAGE, SDS-PAGE, STS-PAGE, SSR and RFLP, indicate that T. petropavlovskyi is genetically distinct from other Chinese endemic wheat landraces [5,59].

Our ML and BI study of the Pgk-1 gene indicates that the A and D genomes of T. petropavlovskyi are basically shared with T. spelta, $T$. compactum and T. sphaerococcum. When the B genome is considered, T. petropavlovskyi groups in one subclade, comparatively distantly related to T. aestivum. In addition, the B-type of $\mathrm{MJ}$ network shows that the accessions of $T$. petropavlovskyi (subgroup III) are distinct from those of other species. SHW-DPW, a synthetic hexaploid wheat with both genomes of T. polonicum and Ae. tauschii [26], based on the $P g k-1$ gene is characterized by the A, B and D genomes, the SHW-DPW is distant from those of T. petropavlovskyi in B and D genomes.

\section{Relationships between T. petropavlovskyi and the tetraploid wheats}

Morphologically, the spikelet of $\mathcal{T}$. petropavlovskyi are similar to those of $\mathcal{T}$. turanicum and $\mathcal{T}$. polonicum [7,26]. Moreover, the cytology of interspecific hybrids between T. petropavlovskyi and tetraploid wheats support a closer relationship with the AABB genomes, compared to wheats with the AAGG genome [2]. According to Akond and Watanabe [24], T. petropavlovskyi is more closely related to $\mathcal{T}$. polonicum than to $T$. durum or $\mathcal{T}$. turgidum. However, Arbuzova et al. [60] and Efremova et al. [18] report that the genes supporting the elongated glumes in T. polonicum and T. petropavlovskyi are not allelic.

In the present study, based on the ML and BI analyses of the genome A Pgk-1 gene, two accessions of $\mathcal{T}$. polonicum have a common topology with T. petropavlovskyi, while the TCS analysis of exon data indicates that the haplotype of A genome in $T$. petropavlovskyi (AS360) is more closely related to T. carthilicum than to other wheats. The phylogenetic analyses and MJ network specific for the $\mathrm{B}$ genome shows that three accessions of $T$. petropavlovskyi group together, and are topologically distant from those of tetraploid wheats. This finding indicates that the B genome of $T$. petropavlovskyi diverge from the one of tetraploid species. Yao et al. [3] and Chen et al. [23] also recognized cytologically that the B genome of $\mathcal{T}$. petropavlovskyi was different from those of hexaploid wheats.

\section{Relationships between T. petropavlovskyi and Ae. tauschii}

Based on RFLPs, Ward et al. [5] found that T. petropavlovskyi is genetically more closely related to accessions of Ae. tauschii from Iran than from China. Yang et al. [10] concluded that $T$. petropavlovskyi is derived from a hybrid between Ae. tauschii and a presumed T. polonicum genotype. However, the phylogenetic analysis of the $A c c-1$ genes indicates that the $\mathrm{D}$ genome of $\mathcal{T}$. petropavlovskyi is very similar to the $\mathrm{D}$ genome orthologs of $\mathcal{T}$. aestivum and only distantly related to Ae. tauschii [26]. In the present study, D genomes of Ae. tauschii belong to two different clusters. One groups with SHW-DPW, T. compactum and T. aestivum cv. Chuannong-16, while T. petropavlovskyi, T. macha, T. spelta, T. sphaerococcum and three Chinese endemic wheats are included in a clade with Ae. tauschii (TA1691). Based on TCS analysis, T. petropavlovskyi shares common topologies with the hexaploid species D genomes. The sequence of the Pgk-1 gene from TA1691 is significantly different from those of other accessions of Ae. tauschii, in agreement with Huang et al. [31]. Together, available results supports that the D genome of $T$. petropavlovskyi is similar to D genome orthologs of $T$. aestivum and only distantly related to $A e$. tauschii.

\section{The divergence time of the T. petropavlovskyi}

Huang et al. [31] report that the diploid progenitors of the A, B and $\mathrm{D}$ genomes present in diploid, tetraploid, and hexaploid wheats radiated between 2.5 and 4.5 MYA. We report that the divergence time of the $\mathrm{A}, \mathrm{B}$ and $\mathrm{D}$ genomes corresponds to 2.61 (95\% C.I., 1.77-3.55), 2.27 (95\% C.I., 1.69-3.19) and 2.05 MYA (95\% C.I., 1.40-2.81), respectively. The divergence of the B genome of T. petropavlovskyi from those of other wheats is dated in this paper is from 0.16 (95\% C.I., $0-0.38)$ to 0.69 MYA (95\% C.I., 0.55-0.70), the earliest date for the four Chinese endemic wheat landraces we considered.

Concerning the $\mathrm{A}$ and $\mathrm{D}$ genome of $\mathcal{T}$. petropavlovskyi, the resulting divergence times are $0.14-0.33$ and $0.11-0.27$ MYA, respectively, values similar to those of other hexaploid species. The divergence time of A genome of T. petropavlovskyi from T. polonicum varies from 0.68 (95\% C.I., 0.41-0.71) to 0.90 MYA (95\% C.I., 0.45-1.41), a divergence earlier than those between T. petropavlovskyi and hexaploid species. The divergence time results indicate that $T$. polonicum may have played a role in the evolutionary history of T. petropavlovskyi.

\section{The possible origin of $T$. petropavlovskyi}

This study shows that the $P g k-1$ sequences of the A genome of T. petropavlovskyi group with T. polonicum. For the Pgk-1 locus of the D genome, the accessions of Ae. tauschii just cluster with the 
T. petropavlovskyi

T. polonicum

synthetic hexaploid wheat

Ae. tauschii
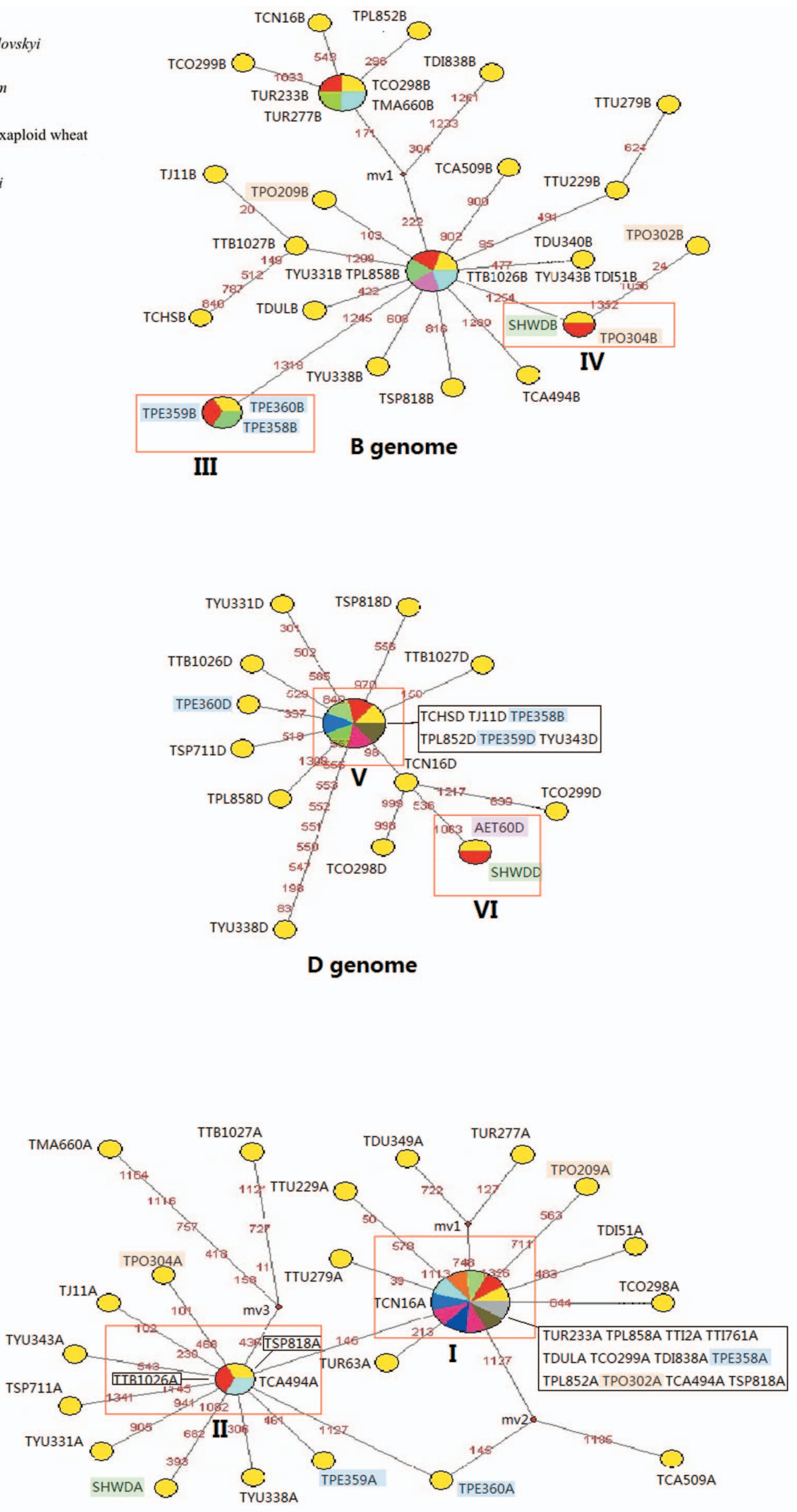

A genome 
Figure 5. Median-joining (MJ) network inferred from the exon+intron sequences of the Pgk-1 gene of T. petropavlovskyi and its related species. Abbreviations of the species names in the $\mathrm{MJ}$ network are listed in Table 1. Haplotypes in the network are represented by circles. Distance between nodes is proportional to the number of nucleotide substitutions among sequences. doi:10.1371/journal.pone.0071139.g005

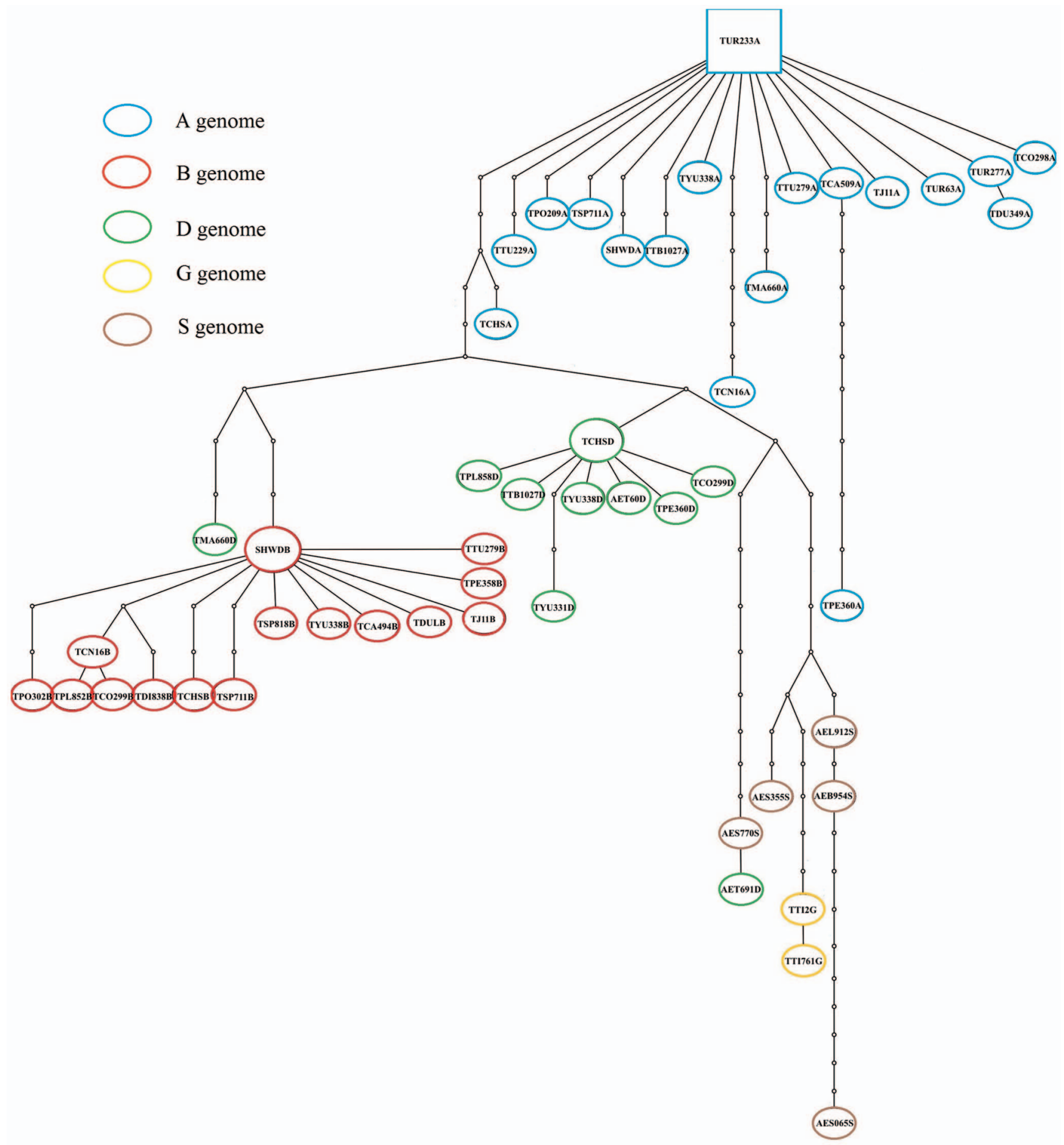

Figure 6. TCS network inferred from the exon sequences of the Pgk-1 gene of $T$. petropavlovskyi and its related species. Abbreviations of species names are listed in Table 1. Haplotypes in the network are represented by circles of different color corresponding to the genomes indicated.

doi:10.1371/journal.pone.0071139.g006 


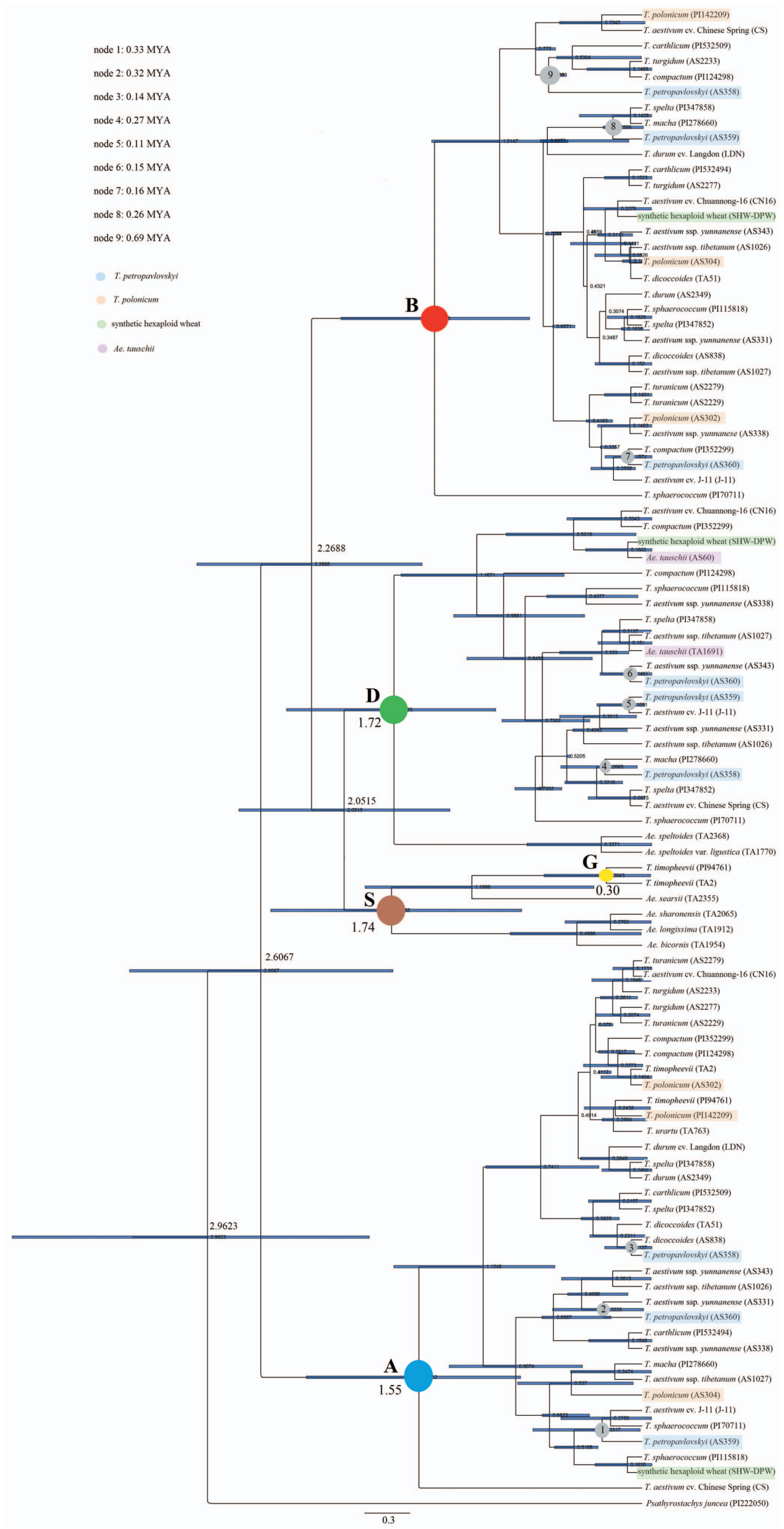


Figure 7. Time-calibrated tree based on intron region of the Pgk-1 sequence of $T$. petropavlovskyi and related species using a Bayesian relaxed BEAST clock method. The different color of node labeled the genome information of the subclade. Numbers at nodes provide the estimated divergence dates. doi:10.1371/journal.pone.0071139.g007

amphiploid SHW-DPW. The Pgk-1 B genome data indicate that T. petropavlovskyi is distantly related to the other three Chinese endemic wheat landraces. The MJ network results are congruent with the results reported above. Also the TCS analysis supports the conclusion that the relationship among haplotypes of $T$. petropavlovskyi and T. polonicum have very similar A and B genomes. We report a distant relationship between T. petropavlovskyi and Ae. tauschii. We conclude that T. petropavlovskyi is neither derived from an independent allopolyploidization event nor from a single mutation in $T$. aestivum. It is most likely that $T$. petropavlorskyi has an origin starting with a natural cross between $\mathcal{T}$. aestivum and $\mathcal{T}$. polonicum, with that event taking place around 0.78 MYA.

\section{References}

1. Yen C, Yang JL, Luo MC (1988) The origin of the Tibetan weedrace of hexaploid wheat, Chinese Spring, Chengdu guangtou and other landraces of white wheat complex from china. In Proceedings of the $7^{\text {th }}$ International Wheat Genetics Symposium Miller TE, Koebner RMD, eds. Cambridge, UK. pp 175179 .

2. Chen Q, Sun YZ, Dong YS (1985) Cytogenetical studies on interspecific hybrids of Xinjiang wheat. Acta Agron Sin 11: 23-28.

3. Yao JX (1983) Research on a new species in Triticum-Xinjiang wheat with ricelike spike. Hereditas (Beijing) 5: 17-20.

4. Kim HS, Ward RW (2000) Pattern of RFLP-based genetic diversity in germplasm pools of common wheat with different geographical or breeding program origins. Euphytica 115: 197-208.

5. Ward RW, Yang ZL, Kim HS, Yen C (1998) Comparative analyses of RFLP diversity in landraces of Triticum aestivum and collections of Aegilops tauschii from China and South Asia. Theor Appl Genet 96: 312-318.

6. Jakubtsiner MM (1959) K poznaniyu pshenits Kitaja/A contirbution to the knowledge of the wheats of China. Bot J 44: 1425-1436.

7. Udaczin RA, Miguschova EF (1970) Novoe v poznanii roda Triticum L.. Venstnik S-Kh Nauki 9: 20-24.

8. Riley R, Coucoli H, Chapman V (1967) Chromosomal interchanges and the phylogeny of wheat. Heredity 22: 233-247.

9. Shao QQ Li CS, Basang CR (1980) Semi-wild wheat from Xizang (Tibet). Acta Genet Sin 7: 150-156.

10. Yang WY, Yen C, Yang JL (1992) Cytogenetic study on the origin of some special Chinese landraces of common wheat. Wheat Inform Serv 75: 14-20.

11. Dorofeev VF, Filatenko AA, Migushova EF, Udaczinz RA, Jakubziner MM (1979) Flora of Cultivated plants. In Wheat Dorofeev VF, Migushova EKolos, L., eds. pp 1-384.

12. Watanabe N, Imamura I (2002) The inheritance and chromosomal location of a gene for long glume phenotype in Triticum petropavlovskyi Udacz. et Migusch. J Genet Breed 57: 221-227.

13. Watanabe N, Sekiya T, Sugiyama K, Yamagishi Y, Imamura I (2002) Telosomic mapping of the homoeologous genes for the long glume phenotype in tetraploid wheat. Euphytica 128: 129-134.

14. Wang HY, Huang XQ Röder MS, Börner A (2002) Genetic mapping of loci determining long glumes in the genus Triticum. Euphytica 123: 287-293.

15. Yen G, Yang JL, Liu XD, Li LR (1983) The distribution of Aegilops tauschii Cosson in China with reference of the origin of the Chinese common wheat. In Proceedings of the $7^{\text {th }}$ International Wheat Genetics Symposium, Sakamoto S, eds. Kyoto, Japan. pp 55-58.

16. Yang RW, Zhou YH, Zheng YL (2001) Analysis on chromosome G-banding of dwarf polish wheat (Triticum polonicum). J Sichuan Agric Univ 19: 112-114.

17. Liu GX, Zhou YH, Zheng YL, Yang RW, Ding CB (2002) Morphological and cytological studies of dwarfing polish wheat (Triticum turgidum concv. polonicum) from Xinjiang, China. J Sichuan Agric Univ 20: 189-193.

18. Efremova TT, Maystrenko OI, Laikova LI, Arbuzova VS, Popova OM (2000) Comparative genetic analysis of hexaploid wheats Triticum petropavlovskyi Udasz. et Migusch. and Triticum aestivum L. Russ J Genet 36: 1142-1148.

19. Yang RW, Zhou YH, Zheng YL, Hu C (2000) Genetic differences and the relationship of gliadin between Triticum polonicum and Triticum petropavlovskyi. J Triticeae Crops 20: 1-5.

20. Chen QF (1999) Discussion on origin of Chinese endemic wheat. Guizhou Agri Sci 27: 20-25

21. Goncharov NP (2005) Comparative genetic analysis-a base for wheat taxonomy revision. Czech J Genet Plant Breed 41: 52-55.

22. Akond ASMGM, Watanabe N, Furuta Y (2008) Comparative genetic diversity of Triticum aestivum-Triticum polonicum introgression lines with long glume and

\section{Acknowledgments}

We thank Dr. Norman Ellstrand, University of California Riverside, for his help in English polishing. We also thank two anonymous reviewers for their very useful comments on this manuscript.

\section{Author Contributions}

Conceived and designed the experiments: QC HYK YHZ. Performed the experiments: QC YW MYZ. Analyzed the data: XF LNS JZ. Contributed reagents/materials/analysis tools: HQZ RWY LZ CBD LLX. Wrote the paper: QC HYK YHZ.
Triticum petropavlovskyi by AFLP-based assessment. Genet Resour Crop Evol 55: 133-141.

23. Chen PD, Liu DJ, Pei GZ, Oi LL, Huang L (1988) The chromosome constitution of three endemic hexaploid wheats in western China. In Proceedings of the $7^{\text {th }}$. International Wheat Genetics Symposium Miller TE, Koebner RMD, eds. Cambridge, UK. pp 75-80.

24. Akond ASMGM, Watanabe N (2005) Genetic variation among Portuguese landraces of 'Arrancada' wheat and Triticum petropavlovskyi by AFLP-based assessment. Genet Resour Crop Evol 52: 619-628.

25. Kang HY, Wang Y, Yuan HJ, Jiang Y, Zhou YH (2008) A new synthesized 6xwheats, derived from dwarfing polish wheat (Triticum polonicum L.) and Aegilops tauschii Cosson. Intern J Agric Res 3: 252-260.

26. Kang HY, Fan X, Zhang HO, Sha LN, Sun GL, et al. (2010) The origin of Triticum petropavlovskyi Udacz. et Migusch.: demonstration of the utility of the genes encoding plastid acetyl-CoA carboxylase sequence. Mol Breed 25: 381395.

27. Sang T (2002) Utility of low-copy nuclear gene sequences in plant phylogenetics. Crit Rev Biochem Mol Biol 37: 121-147.

28. Smith J, Funke M, Woo V (2006) A duplication of gcyc predates divergence within tribe Coronanthereae (Gesneriaceae): phylogenetic analysis and evolution. Plant Syst Evol 261: 245-256.

29. Chalupska D, Lee HY, Faris JD, Evrard A, Chalhoub B, et al. (2008) Acc homoeoloci and the evolution of wheat genomes. Proc Natl Acad Sci 105: 96919696.

30. Huang SX, Sirikhachornkit A, Faris JD, Su XJ, Gill BS, et al. (2002) Phylogenetic analysis of the acetyl-CoA carboxylase and 3-phosphoglycerate kinase loci in wheat and other grasses. Plant Mol Biol 48: 805-820.

31. Huang SX, Sirikhachornkit A, Su XJ, Fairs J, Gill BS, et al. (2002) Genes encoding plastid acetyl-CoA carboxylase and 3-phosphoglycerate kinase of the Triticum/Aegilops complex and the evolutionary history of polyploid wheat. Proc Natl Acad Sci 99: 8133-8138.

32. Golovnina KA, Glushkov SA, Blinov AG, Mayorov VI, Adkison LR, et al. (2007) Molecular phylogeny of the genus Triticum. Plant Syst Evol 264: 195-216.

33. Yan C, Sun GL, Sun DF (2011) Distinct origin of the Y and St genome in Elymus species: evidence from the analysis of a large sample of St genome species using two nuclear genes. PLoS ONE 6: e26853.

34. Wu F, Mueller LA, Crouzillat D, Pétiard V, Tanksley SD (2006) Combining bioinformatics and phylogenetics to identify large sets of single-copy orthologous genes (COSII) for comparative, evolutionary and systematic studies: a test case in the Euasterid plant clade. Genetics 174: 1407-1420.

35. Sun GL, Ni Y, Daley T (2008) Molecular phylogeny of RPB2 gene reveals multiple origin, geographic differentiation of $\mathrm{H}$ genome, and the relationship of the Y genome to other genomes in Elymus species. Mol Phylogenet Evol 46: 897907.

36. Sun GL, Salomon B (2009) Molecular evolution and origin of tetraploid Elymus species. Breed Sci 59: 487-491.

37. Petersen G, Seberg O, Yde M, Berthelsen K (2006) Phylogenetic relationships of Triticum and Aegilops and evidence for the origin of the A, B, and D genomes of common wheat (Triticum aestivum). Mol Phylogenet Evol 39: 70-82.

38. Fan X, Sha LN, Zeng J, Kang HY, Zhang HO, et al. (2012) Evolutionary dynamics of the $P g k-1$ gene in the polyploid genus Kengyilia (Triticeae: Poaceae) and its diploid relatives. PLoS ONE 7: e31122.

39. Gornicki P, Faris J, King I, Podkowinski J, Gill BS, et al. (1997) Plastid-localized acetyl-CoA carboxylase of bread wheat is encoded by a single gene on each of the three ancestral chromosome sets. Poc Natl Acad Sci 94: 14179-14184.

40. Kilian B, Özkan H, Deusch O, Effgen S, Brandolini A, et al. (2007) Independent wheat B and $\mathrm{G}$ genome origins in outcrossing Aegilops progenitor haplotypes. Mol Biol Evol 24: 217-227. 
41. Doyle JJ, Doyle JL (1990) Isolation of plant DNA from fresh tissue. Focus 12: 13-15.

42. Doyle JJ, Doyle JL (1999) Nuclear protein-coding genes in phylogeny reconstruction and homology assessment: some examples from Leguminosae. In The Molecular Systematics and Plant Evolution Hollingsworth PM, Bateman RM, Gornall RJ, eds. Taylor and Francis, London. pp 229-254.

43. Fan X, Sha LN, Yang RW, Zhang HQ, Kang HY, et al. (2009) Phylogeny and evolutionary history of Leymus (Triticeae; Poaceae) based on a single-copy nuclear gene encoding plastid acetyl-CoA carboxylase. BMC Evol Biol 9: 247.

44. Thompson JD, Plewniak F, Poch O (1999) A comprehensive comparison of multiple sequence alignment programs. Nucleic Acids Res 27: 2682-2690.

45. Tamura K, Peterson D, Peterson N, Stecher G, Nei M, et al. (2011) MEGA5: molecular evolutionary genetics analysis using maximum likelihood, evolutionary distance, and maximum parsimony methods. Mol Biol Evol 28: 2731-2739.

46. Posada D, Crandall KA (1998) Modeltest: testing the model of DNA substitution. Bioinformatics 14: 817-818.

47. Felsenstein J (1985) Confidence limits on phylogenies: an approach using the bootstrap. Evolution 39: 783-791.

48. Huelsenbeck JP, Ronquist F (2001) MrBayes: Bayesian inference of phylogenetic trees. Bioinformatics 17: 754-755.

49. Allaby RG, Brown TA (2001) Network analysis provides insights into evolution of 5S rDNA arrays in Triticum and Aegilops. Genetics 157: 1331-1341.

50. Wang HY, Wang XE, Chen PD, Liu DJ (2007) Assessment of genetic diversity of Yunnan, Tibetan, and Xinjiang wheat using SSR markers. J Genet Genomics 34: 623-633.

51. Bordbar F, Rahiminejad MR, Saeidi H, Blattner FR (2011) Phylogeny and genetic diversity of D-genome species of Aegilops and Triticum (Triticeae, Poaceae) from Iran based on microsatellites, ITS, and trnL-F. Plant. Syst Evol 291: 117131.

52. Yan C, Sun GL (2011) Nucleotide divergence and genetic relationships of Pseudoroegneria species. Biochem Syst Ecol 39: 309-319.

53. Huson DH, Bryant D (2006) Application of phylogenetic networks in evolutionary studies. Mol Biol Evol 23: 254-267.

54. Clement M, Posada D, Crandall KA (2000) TCS: a computer program to estimate gene genealogies. Mol Ecol 9: 1657-1659.

55. Drummond AJ, Suchard MA, Xie D, Rambaut A (2012) Bayesian phylogenetics with BEAUti and the BEAST 1.7. Mol Biol Evol 29: 1969-1973.

56. Gaut BS (1998) Molecular clocks and nucleotide substitution rates in higher plants. In The Evolutionary Biology Hecht MK, ed. Plenum Press New York, .pp 93-120.

57. Wolfe KH, Gouy M, Yang YW, Sharp PM, Li WH (1989) Date of the monocotdicot divergence estimated from chloroplast DNA sequence data. Proc Natl Acad Sci 86: 6201-6205.

58. Wolfe KH, Li WH, Sharp PM (1987) Rates of nucleotide substitution vary greatly among plant mitochondrial, chloroplast, and nuclear DNAs. Proc Natl Acad Sci 84: 9054-9058.

59. Wei YM, Zheng YL, Liu DC, Zhou YH, Lan XJ (2002) HMW-glutenin and gliadin variations in Tibetan weedrace, Xinjiang rice wheat and Yunnan hulled wheat. Genet Resour Crop Evol 49: 327-330.

60. Arbuzova V S, Efremova TT, Laikova LI, Maystrenko OI, Popova OM, et al. (1996) The development of precise genetic stocks in two wheat cultivars and their use in genetic analysis. Euphytica 89: 11-15. 